\title{
Resonant-to-nonresonant transition in electrostatic ion-cyclotron wave phase velocity
}

\author{
J. J. Carroll III ${ }^{1,2}$, M. E. Koepke ${ }^{1}$, M. W. Zintl ${ }^{1,3}$, and V. Gavrishchaka ${ }^{4}$ \\ ${ }^{1}$ Department of Physics, West Virginia University, Morgantown, WV 26506-6315, USA \\ ${ }^{2}$ present address: Eastern Michigan University, Ypsilanti, MI 48197, USA \\ ${ }^{3}$ present address: SARA, Inc., Huntington Beach, CA 92649, USA \\ ${ }^{4}$ Science Applications International Corp., McLean, VA 22102, USA
}

Received: 2 January 2002 - Revised: 19 April 2002 - Accepted: 6 June 2002

\begin{abstract}
Because of the implications for plasmas in the laboratory and in space, attention has been drawn to inhomogeneous energy-density driven (IEDD) waves that are sustained by velocity-shear-induced inhomogeneity in crossfield plasma flow. These waves have a frequency $\omega_{r}$ in the lab frame within an order of magnitude of the ion gyrofrequency $\omega_{c i}$, propagate nearly perpendicular to the magnetic field $\left(k_{z} / k_{\perp} \ll 1\right)$, and can be Landau resonant $\left(0<\omega_{1} / k_{z}<v_{d}\right)$ with a parallel drifting electron population (drift speed $v_{d}$ ), where subscripts 1 and $r$ indicate frequency in the frame of flowing ions and in the lab frame, respectively, and $k_{z}$ is the parallel component of the wavevector. A transition in phase velocity from $0<\omega_{1} / k_{z}<v_{d}$ to $0>\omega_{1} / k_{z}>v_{d}$ for a pair of IEDD eigenmodes is observed as the degree of inhomogeneity in the transverse $\boldsymbol{E} \times \boldsymbol{B}$ flow is increased in a magnetized plasma column. For weaker velocity shear, both eigenmodes are dissipative, i.e. in Landau resonance, with $k_{z} v_{d}>0$. For stronger shear, both eigenmodes become reactive, with one's wavevector component $k_{z}$ remaining parallel, but with $\omega_{1} / k_{z}>v_{d}$, and the other's wavevector component $k_{z}$ becoming anti-parallel, so that $0>\omega_{1} / k_{z}$. For both eigenmodes, the transition (1) involves a small frequency shift and (2) does not involve a sign change in the wave energy density, which is proportional to $\omega_{r} \omega_{1}$, both of which are previously unrecognized aspects of inhomogeneous energydensity driven waves.
\end{abstract}

\section{Introduction}

In recent experiments on Inhomogeneous Energy-Density Driven (IEDD) waves (Ganguli et al., 1985; 1988; 1989; Koepke et al., 1994; Amatucci et al., 1996; Gavrishchaka et al., 1996), quantitative comparisons with theoretical predictions were obtained (Koepke et al., 1995; 1998a; Amatucci et al., 1998; Carroll et al., 1998; Peñano et al., 1998). Beyond verifying the existence of the excitation mechanism,

Correspondence to: M. E. Koepke (mkoepke@wvu.edu) these comparisons reinforced detailed aspects of the theoretical model and also stimulated refinements and an expanded awareness of the details of the model. This new knowledge of how perpendicular-velocity shear substantially changes plasma stability is being used to interpret recent observations of extremely low frequency waves in the auroral zone of the ionosphere and magnetosphere (Kintner et al., 1996; 2000a; 2000b; Bonnell et al., 1996; Bonnell, 1997; Lund et al., 1999; Hamrin et al., 2001; André et al., 2002). Here, we report on aspects of the dispersion relation heretofore unpredicted in the model.

Our work concentrates on IEDD waves, which are sustained by velocity-shear-induced inhomogeneity in the wave energy density. By creating in a magnetized plasma column a localized region of $\boldsymbol{E} \times \boldsymbol{B}$ flow, we generate a perpendicularflow layer in which the electrons and ions drift together azimuthally with a common speed $\nu_{E}$. For $E_{r}>0$ and $B_{z}>0$, we have $v_{\theta}=-v_{E}$. Here, "localized" means that the region of elevated perpendicular drift velocity is narrow enough to be considered separated from both the cylindrical axis and the plasma's radial edge. Electrostatic IEDD waves propagate primarily in the $\boldsymbol{E} \times \boldsymbol{B}$ direction, have frequencies in a wide range near the ion gyrofrequency, and are excited in the cylindrical layer of perpendicular flow (Koepke et al., 1998b). In space, IEDD waves are expected to grow at the edges of auroral arcs where strong, small-scale electric fields are found (Kelley and Carlson, 1977; Marklund et al., 1994; Moore et al., 1996). Since it is a nonlocal mode, there is a Doppler shift of $\Delta \omega=\left|k_{\theta} v_{E}\right|$ between the real part of the dispersion relation's eigenfrequency $\omega_{r}$, which is influenced by the entire velocity profile (i.e. not just the portion where the shear is maximum), and the effective mode frequency $\omega_{1}$ in the frame of the $\boldsymbol{E} \times \boldsymbol{B}$ drift. The radial wavepacket that typically fills the localized flow layer consists of multiple unstable eigenstates, each identifiable in the spectra of frequency (Koepke et al., 1998a). If a parallel electron drift is present, some eigenmodes get Landau damped and some Landau grow, but all have characteristics sensitive to the velocity-shear parameters. 
The significance of ion-cyclotron waves lies in the effective particle acceleration resulting from the gyroresonant nature of the interaction between these waves and the ions in magnetized space plasma (Kintner et al., 1978; Mozer et al., 1980). In the auroral acceleration zone, gyro-resonant waveparticle interactions are believed to be responsible for the unexpectedly large fraction of heavy ions of ionospheric origin in the magnetosphere (Pollock et al., 1990).

Electrostatic ion-cyclotron (EIC) waves in the presence of parallel electron current have been observed with a rocket traversing an auroral arc (Bering, 1984). Ion beams and density gradients were ruled out as possible sources of free energy for the waves. Although the parallel current density, inferred from magnetometer data, was $5 \mu \mathrm{A} / \mathrm{m}^{2}$, the ratio of the electron drift speed to the electron thermal speed was approximately 0.002 (for $10^{5} \mathrm{~cm}^{-3}$ plasma density and $0.1 \mathrm{eV}$ electron temperature), far below the EIC-wave excitation threshold in the absence of velocity shear. The possibility that the current was carried by only a small fraction (less than $2 \%$ ) of the electron population was considered since, if fewer electrons carry the current, the estimated drift velocity would approach the critical value for excitation. Perpendicular electric fields ( $E_{\perp} \approx 7 \mathrm{mV} / \mathrm{m}$ ) were also observed (Robinson et al., 1981) which may have contributed to the destabilization of the waves.

Simultaneous observations of electrostatic hydrogencyclotron waves and $\mathrm{keV}$ upstreaming ions were made with the S3-3 satellite (Kintner et al., 1979). Electron drift was considered as the possible driving mechanism for the waves. However, the S3-3 magnetometer data indicated that the parallel current density was $0.2 \mathrm{~A} / \mathrm{m}^{2}$, approximately $50 \%$ of the estimated excitation threshold current density for EIC waves in the absence of velocity shear. It was explained that this discrepancy could be partially removed since the measurement was averaged over a $300 \mathrm{~km}$ path length while the cyclotron waves were observed in two bursts, each corresponding to a $90 \mathrm{~km}$ path length. Numerous examples of perpendicular electrostatic shocks containing electrostatic ion-cyclotron turbulence were seen by the S3-3 satellite at altitudes of $1000-8000 \mathrm{~km}$ with scale sizes ranging from $200 \mathrm{~m}$ to $10 \mathrm{~km}$ (Torbert and Mozer, 1978). Shocks with smaller scale sizes outnumbered those with large scale sizes. They define electrostatic shocks as "confined spatial regions in which extremely large, dc electric fields are observed, and in which abrupt discontinuities in plasma parameters, particle fluxes, and waves properties often occur." The shocks were typically bordered by very-low-frequency (VLF) emitting regions, and often occurred in regions of intense parallel, $100 \mathrm{eV}$ electron streams.

Sounding-rocket observations of broadband electrostatic ion-cyclotron waves at the edge of an auroral arc in the presence of perpendicular-velocity shear and magnetic-fieldaligned electron current have been reported by Kelley and Carlson (1977). The relationship between the strong shear and intense irregularities indicated that a velocity-shear mechanism, unknown at the time of the original analysis operated at wavelengths short in comparison with scale lengths associated with the Kelvin-Helmholtz instability. Ganguli et al. (1994) discuss a two-step process whereby large-scale Kelvin-Helmholtz waves steepen nonlinearly to give rise to localized vortical structures in electrostatic potential in which IEDD waves can grow.

With the advent of higher sampling rates for rocket instrumentation, attention has been turned to using IEDD waves for interpreting observations dynamically intense geomagnetic activity at the highly structured edges of auroral arcs. The ARCS-4 rocket detected sheared plasma flow, ion heating, and ion-cyclotron waves in an auroral arc (Moore et al., 1996). The superthermal and highly variable plasma winds were consistent with a highly structured dc electric field. The rate of shear in the perpendicular plasma flow associated with these structures was on the order of 5\% of the oxygen gyrofrequency, large enough for the excitation and subsequent dissipation of shear-driven waves to be considered as a primary ion heating mechanism.

Satellite measurements of perpendicular-velocity shear in the auroral ionosphere are prevalent in the literature and many of these reports are able to correlate the shear with thermal-ion upwelling from the topside ionosphere. This correlation would be expected if velocity shear excites waves that heat ions as they flow from the ionosphere to the magnetosphere. Lu et al. (1992) were able to correlate directly the velocity shear with thermal-ion upwelling by making simultaneous observations with the DE- 1 satellite at high altitude $(\geq 12000 \mathrm{~km})$ and the DE-2 satellite at low altitude $(\geq 800 \mathrm{~km})$. DE- 2 , on its own, provided clear indications of broadband fluctuations associated with large velocity shears, parallel electron current (generally well below the anticipated ion-cyclotron wave-excitation threshold in homogeneous plasma), and upward-flowing conic-shaped ion energy distributions in the auroral F-region (Basu et al., 1988). Thermal-ion upwellings were also observed by the Hilat satellite in the dayside polar ionosphere where Tsunoda et al. (1989) report a strong correlation of ion heating with shear in the convective velocity. These authors state that shear in the perpendicular plasma flow is common to all thermal-ion-upwelling observations and discuss the possibility that shear provides a substantial portion of the free energy required to drive the process.

Sounding rocket data from SCIFER and AMICIST provide the best evidence yet of the identity of the waves responsible for auroral ion energization. The SCIFER experiment (Kintner et al., 1996) documented the microphysical signatures of auroral outflow in the cleft-ion fountain, believed to be the principal source of mass for the magnetosphere, especially $\mathrm{O}^{+}$, as the payload passed through several discrete aurora. SCIFER provided continuous spatial/temporal resolution two orders of magnitude better than that achieved by previous orbiting spacecraft and correlated one-to-one the structured perpendicular ion acceleration events with broadband, low-frequency electric fields and plasma density depletions. The AMICIST experiment (Bonnell et al., 1996; Lynch et al., 1996) did the same thing with the nightside auroral oval where the outflow is smaller but the physics appears to be the 


\section{WVU Q-machine}

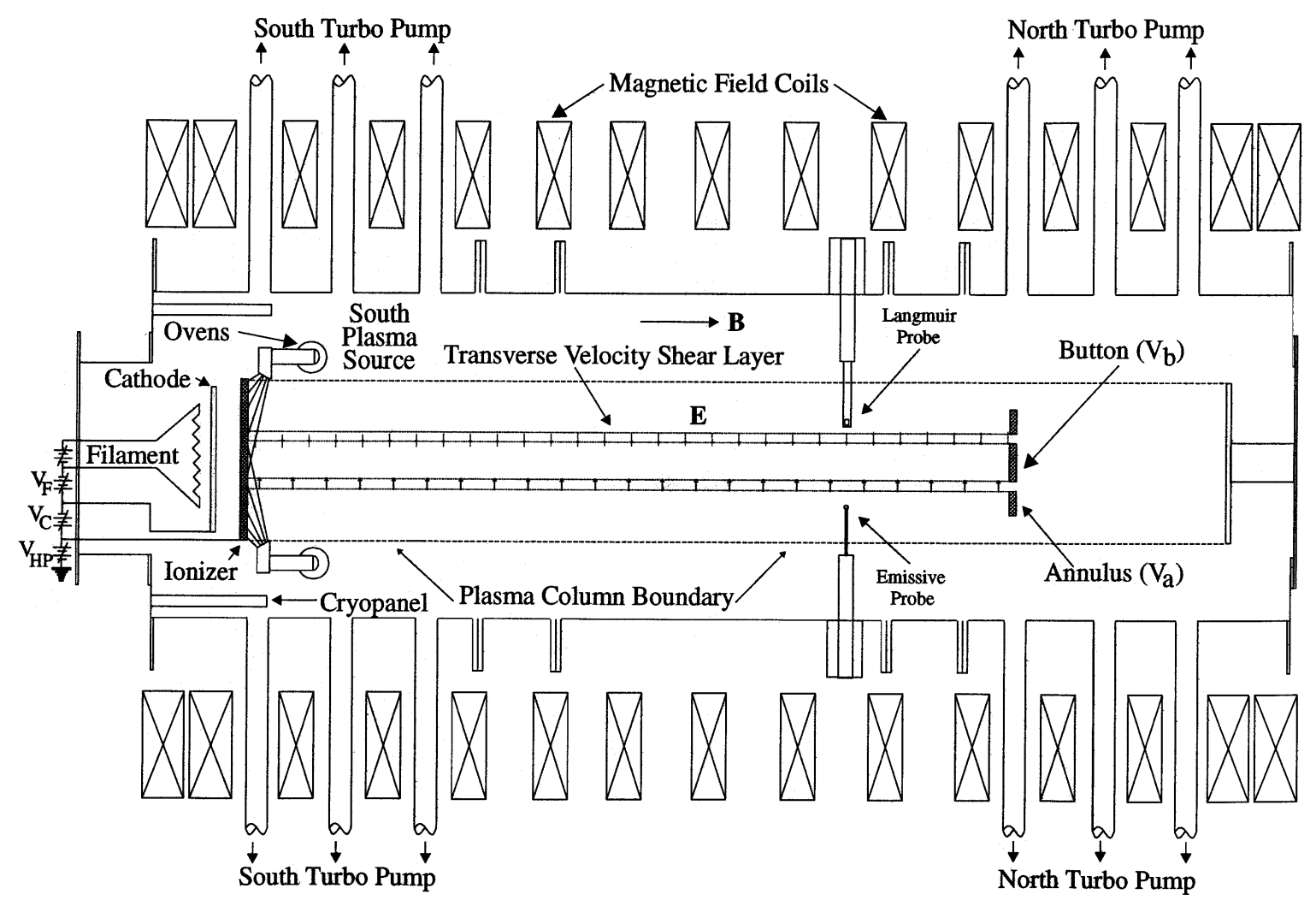

Fig. 1. Diagram of WVU Q Machine.

same. Kintner et al. (1996), Bonnell et al. (1996), and Bonnell (1997) identify IEDD waves as being responsible for the ion acceleration. Bonnell (1997) shows that the wave intensity and the level of velocity shear in the data increase and decrease synchronously, reinforcing the belief that the presence of velocity shear is an essential element of the wave-particle interaction process. Hamrin et al. (2001) compare Freja measurements of BB-ELF waves with laboratory measurements of IEDD waves and conclude that velocity shear plays an important role in the generation mechanism of BB-ELF waves. In this paper, we report on a transition in the nature of the mode, from being predominantly dissipative (i.e. driven by electron current and modified by shear) to being predominantly reactive (i.e. driven by shear and modified by electron current).

\section{Experimental approach}

The West Virginia University (WVU) Q machine (Rynn and D'Angelo, 1960; Koepke et al., 1998b) produces quiescent, steady-state, magnetized ( $0.5 \mathrm{~T}$ maximum, $\boldsymbol{B}=B \hat{z}$ ) plasma (diameter of $6.4 \mathrm{~cm}$ ), with comparable electron and ion temperatures $\left(T_{e} \approx T_{i} \approx 0.2 \mathrm{eV}\right)$, density $n \approx 10^{14}-10^{17} \mathrm{~m}^{-3}$, undisturbed plasma potential $V_{p 0} \approx-2 \mathrm{~V}$, and base neutral pressure $P_{n}<10^{-7} \mathrm{~Pa}$. Plasma density, electron temperature, and electron drifts are measured with current-collecting Langmuir probes, whereas floating emissive probes are used to measure plasma potential.

In the single-source configuration, shown in Fig. 1, a segmented-disk end electrode is used to produce and control the perpendicular plasma flow and the parallel electron drift velocity (Carroll et al., 1994). Typical values achievable with the segmented disk electrode in the WVU Q Machine are $v_{d} / v_{t e}=0.1\left(v_{d} / v_{t i}=20\right)$ and $v_{E} / v_{t e}=0.01\left(v_{E} / v_{t i}=2\right)$, where $v_{t e}\left(v_{t i}\right)$ is the electron (ion) thermal speed. Fluctuations in the current collected by the segmented disk electrode have a spectrum nearly identical to the spectrum associated with fluctuations in ion saturation current collected by a Langmuir probe located at a similar radius anywhere along the $3 \mathrm{~m}$ plasma column.

The disk electrode is made of five coplanar, concentric circular segments heated to prevent surface contamination. The applied voltages $V_{0}, V_{1}, V_{2}, V_{3}$, and $V_{4}$ on the inner button segment $(r \leq 0.80 \mathrm{~cm})$ and on the adjacent annular segments $(0.90 \mathrm{~cm} \leq r \leq 1.35 \mathrm{~cm}, 1.40 \mathrm{~cm} \leq r=1.85 \mathrm{~cm}$, $1.90 \mathrm{~cm} \leq r=2.35 \mathrm{~cm}, 2.40 \mathrm{~cm} \leq r=2.85 \mathrm{~cm}$, respectively, are set with independent power supplies. Here, we bias positively the inner two segments and electrically float the other 


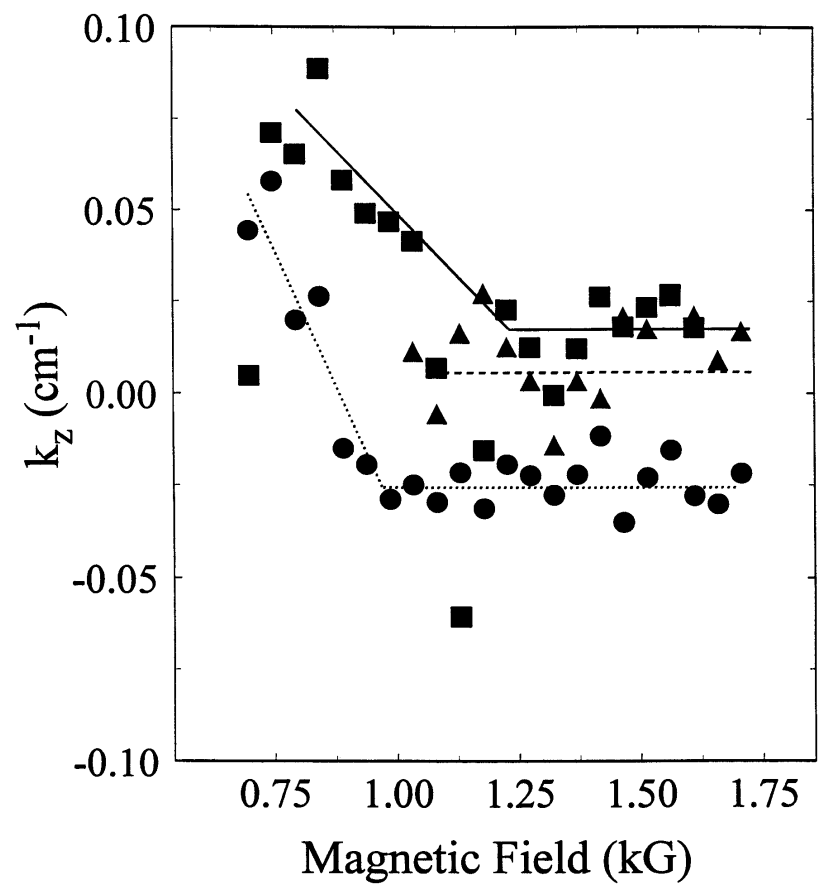

Fig. 2. Transition in $k_{z}$ as $B$ increases. The three lines guide the eye. Square (circle) symbols correspond to the higher-frequency (lowerfrequency) spectral feature. The region of the plot associated with values of $k_{z}<0$ is identified as being in the predominantly reactive regime. The seven square symbols along the top of the solid line and the four circle symbols along the top of the dotted line are identified with the predominantly reactive regime. Other symbols having $k_{z}>0$ are identified with the predominantly dissipative regime. The triangle symbols correspond to a third feature appearing in the wavevector spectrum for magnetic field strengths exceeding $1 \mathrm{kG}$.

segments.

To control the electron drift speed without significantly changing the inhomogeneous transverse-velocity profile, the bias of the hot plate is adjusted from its usual $V=0$ condition. Raising the hot-plate bias while the disk-electrode bias is positively biased reduces the electron drift speed, whereas lowering the hot-plate bias while the disk-electrode is positively biased, increases the electron drift speed. The growth rate $\gamma$ of IEDD waves can be determined for a particular value of drift speed by introducing a step in the parallel electron drift speed from just below the excitation threshold to above the threshold and monitoring the subsequent exponential increase in the wave amplitude (Koepke et al., 1998b). Experiments in a potassium plasma have confirmed that, for dissipatively driven IEDD waves, the growth rate increases with current.

The predicted $v_{\theta}$ dependence of the mode frequency $\omega_{r}$ in the lab frame has been confirmed experimentally using both single-eigenmode and double-eigenmode manifestations of IEDD waves (Koepke et al., 1995; Carroll et al., 1998; Peñano et al., 1998). In these cases, $\omega_{r}$ is less than the doppler-shifted frequency $\omega_{1}$ in the frame of the flow frame, $\omega_{c i}<\omega_{1}<2 \omega_{c i}$, and $\left|\omega_{1}-\omega_{r}\right|$ increases with increasing
$v_{E}$. For the lowest-order radial eigenmode, $\omega_{1}$ is relatively insensitive to $k_{\theta} v_{E}$ so that as $k_{\theta} v_{E}$ increases, $\omega_{r}$ can downshift from above to below $\omega_{c i}$. For the higher radial eigenmodes, $\omega_{1}$ increases more sensitively with increasing $v_{E}$ so that $\omega_{r}$ shifts less with increasing $v_{E}$ (Carroll et al., 1998). Large values of $k_{\theta}$ or $v_{E}$ lead to small values of mode frequency (e.g. $\omega_{r} / \omega_{c i} \approx 0.3$ ). For even larger values of $k_{\theta}$ or $v_{E}, k_{\theta} v_{E}$ can exceed $\omega_{1}$ so that $\omega_{r}$ and $\omega_{1}$ have opposite signs and the observed mode frequency appears to increase with increasing $v_{E}$ (Amatucci et al., 1996). Conceptually, such a frequency upshift is actually a frequency downshift of greater than $100 \%$. The product $\omega_{r} \omega_{1}$ is proportional to the wave energy density and having $\omega_{r} \omega_{1}<0$ in the flow region and $\omega_{r} \omega_{1}>0$ in the no-flow region provides the reactive free energy. In this paper, we show that, for small values of $k_{\theta} v_{i}$, IEDD waves can be reactively excited without $\omega_{r} \omega_{1}<0$ in the flow region.

An array of two Langmuir probes, located at the same radius, is used to measure $k_{\theta}$ and $k_{z}$, the axial and azimuthal components of the mode's wavevector, from the dependence of relative phase (between the two signals collected simultaneously by the probe tips) on the orientation of the probe array with respect to the $z$ direction. For travelling waves, $k_{\theta}=\Delta \phi /\left(r_{p} \Delta \theta\right)$ and $k_{z}=\Delta \phi /(\Delta z)$, where $\Delta \phi$ is the measured phase difference (in radians) between probe signals, $r_{p}$ is the radial position of the probe tips, and $\Delta \theta$ and $\Delta z$ are the azimuthal and axial separation of the probe tips. Two arrays, oriented perpendicular to each other, allowed azimuthal measurements of the phase $\left(r_{p} \Delta \theta=3 \mathrm{~mm}\right)$ and axial measurements of the phase $(\Delta z=6.3 \mathrm{~cm})$ to be acquired simultaneously. This feature improves the assignment of $k_{\theta}$ and $k_{z}$ values to specific spectral features. The halo-tip probe design (Koepke et al., 1999) increases radial resolution for a given signal level and decreases the perturbation to the plasma. This design improvement was motivated by the need for reliable wave propagation measurements in a plasma containing multiple oscillatory eigenmodes that are closely spaced in frequency, share similar three-dimensional mode structure, and are localized in a radially narrow cylindrical shell of transverse flow radially separated from the plasma column edge. The probe's radially narrow collecting area is supported by thin stalks from the main support tube that surrounds the heating element.

The value of $k_{z}$ helps identify the predominance of dissipative or reactive responses for a particular IEDD eigenmode. In contrast to CDEIC waves, IEDD waves are observed with an axial wavelength smaller than the length of the laboratory plasma column in which they exist (Koepke et al., 1994). In the case of multiple IEDD eigenmodes, each eigenmode can have a different axial wavelength. Some may be predominantly dissipative and some may be predominantly reactive. For predominantly dissipative eigenmodes in sodium, the axial component of the propagation is in the direction of the electron drift, with

$$
\begin{aligned}
& \omega_{1} /\left(k_{z} v_{d}\right)=\left(\omega_{r}+k_{\theta} v_{E}\right) /\left(k_{z} v_{d}\right) \\
& \approx\left(1+k_{\theta} \rho_{i} v_{E} / v_{t i}\right) \omega_{c i} /\left(\left(0.08 \mathrm{~cm}^{-1}\right)\left(1 \times 10^{7} \mathrm{~cm} / \mathrm{s}\right)\right)
\end{aligned}
$$



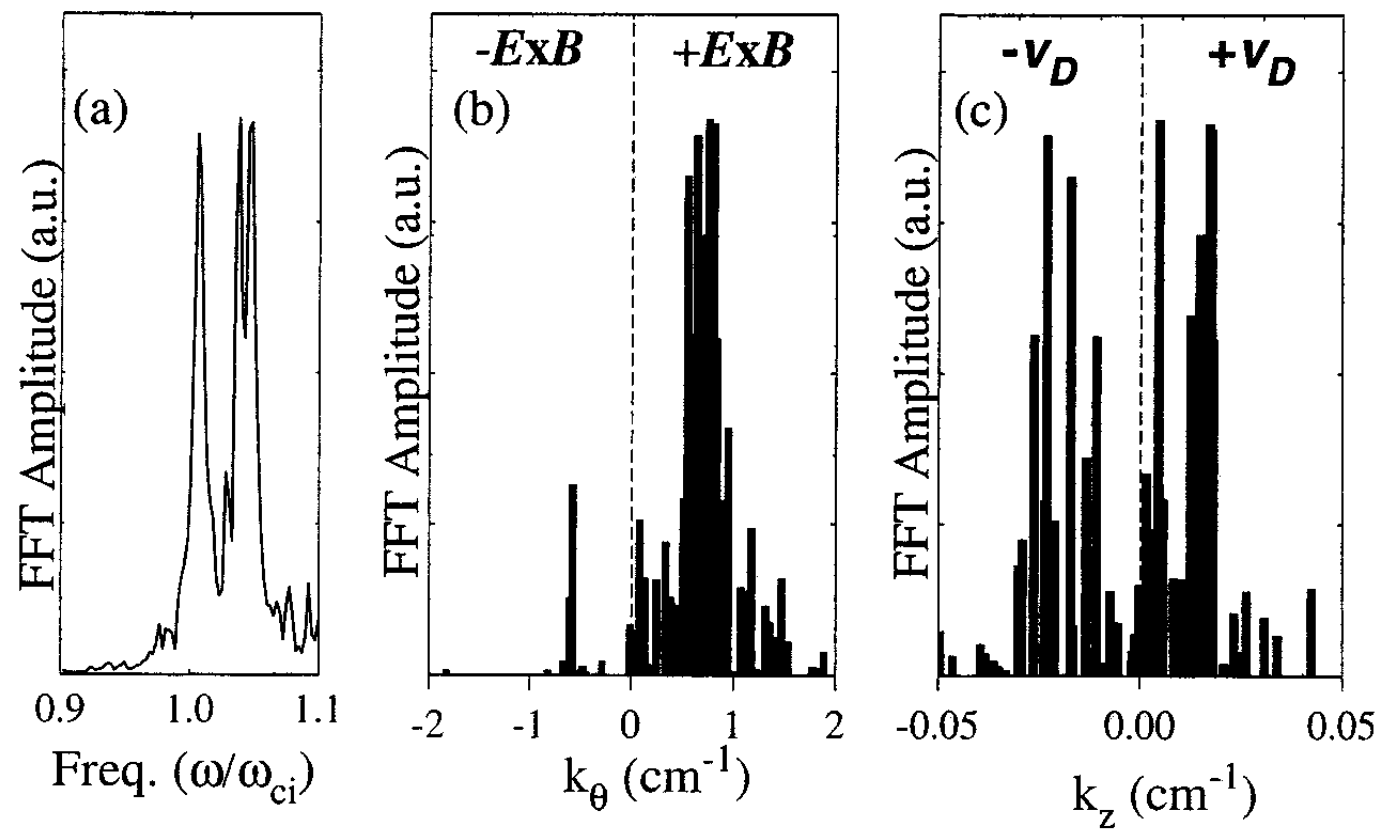

Fig. 3. Probe-acquired spectra of (a) frequency, (b) $k_{\theta}$, and (c) $k_{z}$ for the reactively driven IEDD eigenmodes in Fig. 1 at $B=1.5 \mathrm{kG}$. Note that the values of $k_{z}$ for the two eigenmodes have different signs, indicating counter propagation along $z$.

$$
\begin{aligned}
& \approx\left(1+\left(1.8 \mathrm{~cm}^{-1}\right)(0.24 \mathrm{~cm})(1)(2 \pi)(60 \mathrm{kHz}) /\right. \\
& \left.\left(8 \times 10^{7} \mathrm{~s}^{-1}\right)\right) \\
& \approx 0.7
\end{aligned}
$$

where $k_{z}$ is the parallel component of the wavevector and $v_{t i}$ is the ion thermal speed.

\section{Resonant-to-nonresonant transition in phase velocity}

Figure 2 shows the change in $k_{z}$ for a pair of IEDD eigenmodes as the magnetic field strength is increased. The density is $3 \times 10^{1} 3 \mathrm{~m}^{-3}$, more than an order of magnitude smaller than for our usual IEDD-wave experiments. The bias configuration is $V_{0}=10 \mathrm{~V}$ and $V_{1}=0 \mathrm{~V}$, corresponding to a radially outward electric field. Electric-field measurements indicate a peak value of $6 \mathrm{~V} / \mathrm{cm}$, corresponding to $v_{E}=6 v_{t i}$ at the highest value of $B$. The mode frequency associated with one eigenmode (square symbols in Fig. 2) is approximately $4 \%$ above the ion gyrofrequency at $B=0.8 \mathrm{kG}$ and downshifts (relative to $\omega_{c i}$, which is also changing) to approximately $2 \%$ below the ion gyrofrequency at $B=1.5 \mathrm{kG}$. The second eigenmode (circle symbols in Fig. 2) downshifts from $20 \%$ above to $3 \%$ above the gyrofrequency over the same range of magnetic-field strength. The $k_{z}$ value of the largerfrequency eigenmode shifts from $0.08 \mathrm{~cm}^{-1}$ to $0.03 \mathrm{~cm}^{-1}$ in the first half of the range in $B$. The $k_{z}$ value of the smallerfrequency eigenmode shifts from $0.05 \mathrm{~cm}^{-1}$ to $-0.025 \mathrm{~cm}^{-1}$ in the first third of the range of $B$. The values of $k_{\theta}$ for both eigenmodes are relatively small and essentially the same $\left(k_{\theta}=0.85 \mathrm{~cm}^{-1}\right)$. At the higher values of $B$, a third feature (triangle symbols in Fig. 2) appears at a frequency located between the original two eigenmodes, unlike the dissipative cases (Koepke et al., 1998a), where new eigenmodes always appeared at frequencies higher than the previously existing eigenmodes. In comparing the behavior of these eigenmodes to the dissipative eigenmodes just mentioned, it should be noted that $\left(k_{\theta} \rho_{i}\right)^{2}$ is at least a factor of four smaller in the case reported here.

These changes in $k_{z}$ correspond to changes in phase velocity and thus have consequences for labeling these eigenmodes as predominantly dissipative or reactive. At small $B$, both eigenmodes are Landau resonant with the drifting electrons. That is to say the parallel phase velocity is aligned with and is less than the parallel drift of electrons. At higher $B$, both eigenmodes become non-resonant, with one's wavevector component remaining parallel and the other's component becoming anti-parallel.

A wavenumber spectrum consists of the real (FFT amplitude) and imaginary (FFT phase $\Delta \phi$ ) arrays resulting from a cross-correlation analysis, and is displayed by plotting the former vs. the latter. A line is used to connect the data in the frequency spectrum, whereas each datum in the $k_{\theta}$ spectrum is represented by a bar with height equal to FFT amplitude and horizontal position equal to $k_{\theta}$. As experimental parameters are varied, changes in the dissipative and reactive nature of individual eigenmodes within a group of eigenmodes can be followed conveniently by monitoring such a $k_{\theta}$ spectrum. Figure 3 shows the frequency spectrum, the $k_{\theta}$ spectrum, and the $k_{z}$ spectrum for the two eigenmodes in Fig. 2 at $B=1.5 \mathrm{kG}$, i.e. in the reactive regime.

The parallel phase velocity of the higher-frequency eigen- 


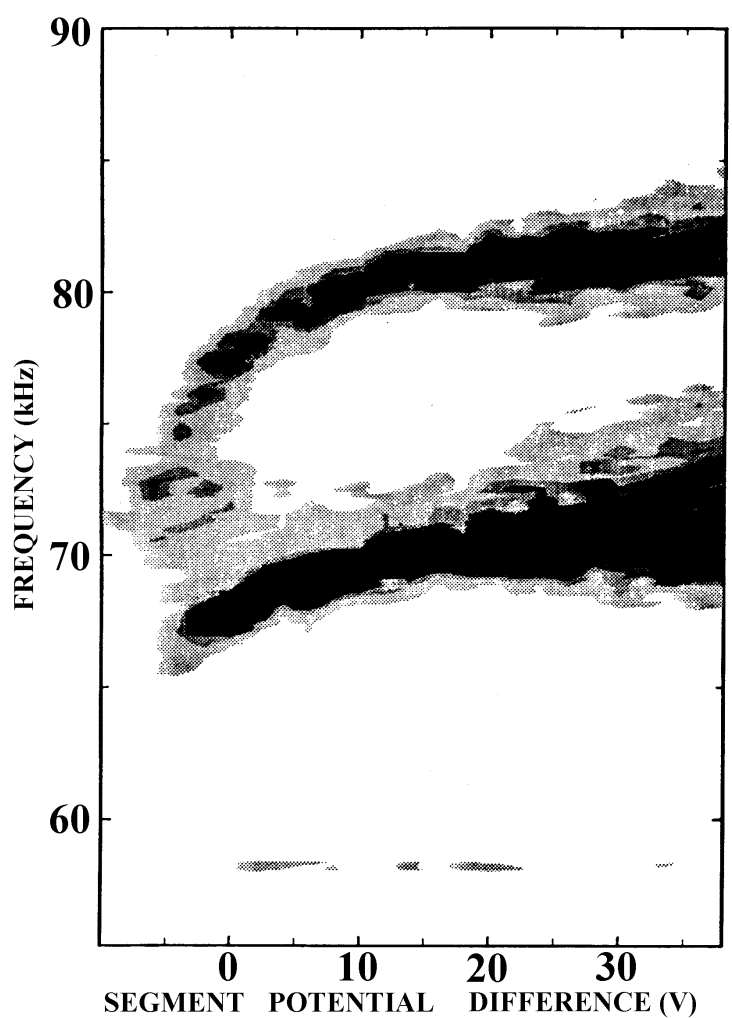

Fig. 4. Contour plot of fluctuation amplitude versus frequency for $0<V_{0}<37.5 \mathrm{~V}$ and $V_{1}=0 \mathrm{~V}$ at $B=1.5 \mathrm{kG}$ as measured on a Langmuir probe in sodium plasma. Each set is an average of 4 realizations. Two spectral features are evident, both upshifting with increasing $V_{0}$. The ion gyrofrequency is $70 \mathrm{kHz}$.

mode is parallel to the electron drift with

$$
\begin{aligned}
& \omega_{1} /\left(k_{z} v_{d}\right)=\left(\omega_{r}+k_{\theta} v_{E}\right) /\left(k_{z} v_{d}\right) \\
& \approx\left(1+k_{\theta} \rho_{i} v_{E} / v_{t i}\right) \omega_{c i} /\left[\left(0.02 \mathrm{~cm}^{-1}\right)\left(1 \times 10^{7} \mathrm{~cm} / \mathrm{s}\right)\right] \\
& \approx\left(1+\left(0.8 \mathrm{~cm}^{-1}\right)(0.15 \mathrm{~cm})(1)(2 \pi)(104 \mathrm{kHz}) /\right. \\
& \left(2 \times 10^{7} \mathrm{~s}^{-1}\right) \\
& \approx 3.5
\end{aligned}
$$

The parallel phase velocity of the lower-frequency eigenmode is antiparallel to the electron drift, with

$$
\begin{aligned}
& \omega_{1} /\left(k_{z} v_{d}\right)=\left(\omega_{r}+k_{\theta} v_{E}\right) /\left(k_{z} v_{d}\right) \\
& \approx\left(1+k_{\theta} \rho_{i} v_{E} / v_{t i}\right) \omega_{c i} /\left(\left(-0.02 \mathrm{~cm}^{-1)}\left(1 \times 10^{7} \mathrm{~cm} / \mathrm{s}\right)\right)\right. \\
& \approx\left(1+\left(0.65 \mathrm{~cm}^{-1}\right)(0.15 \mathrm{~cm})(1)(2 \pi)(100 \mathrm{kHz}) /\right. \\
& \left(-2 \times 10^{7} \mathrm{~s}^{-1)}\right) \\
& \approx-3.5
\end{aligned}
$$

These eigenmodes upshift with increasing $v_{E}$, as shown in Fig. 4. Initially located at $66 \mathrm{kHz}\left(0.94 \omega_{c i}\right)$ and $73 \mathrm{kHz}$ $\left(1.03 \omega_{c i}\right)$, they upshift to $70 \mathrm{kHz}\left(1.00 \omega_{c i}\right)$ and $81 \mathrm{kHz}$ $\left(1.14 \omega_{c i}\right)$ as the bias $V_{0}$ is increased. Electric field measurements indicate values of $6 \mathrm{~V} / \mathrm{cm}$, corresponding to $v_{E}=6 v_{t i}$ at high $V_{0}$.

\section{Discussion}

These eigenmodes survive even when the parallel electron drift drops below $1 \%$ of the electron thermal speed, i.e. when the parallel electron current is essentially eliminated, as expected for waves driven by the reactive response of the IEDD wave-excitation mechanism. Since $\omega / k_{z} v_{d}>1$ for the higher-frequency eigenmode and $\omega / k_{z} v_{d}<0$ for the lowerfrequency eigenmode, inverse Landau damping is not destabilizing the waves. The only other laboratory observation of spontaneously excited, electrostatic ion-cyclotron waves propagating antiparallel to the electron drift direction is the ion-beam-driven waves reported by Hauck et al. (1978), but in this case the wave frequency normalized to the ion gyrofrequency does not upshift with experimental parameters.

The amplitude of these non-resonant eigenmodes is not sensitive to the relative bias between the hot-plate the diskelectrode, as is the amplitude of the dissipative eigenmodes, implying that, as expected for predominantly reactive eigenmodes, they do not tap the free energy available in the parallel electron drift. Consequently, the stepped-bias technique for measuring growth rate is not applicable to the predominantly reactive IEDD eigenmodes and a subcritical value of ionizer bias could not be found.

The small values of $k_{\theta}$ result in small values of $\left(k_{\theta} \rho_{i}\right)^{2} \approx$ 0.04 and small Doppler shifts $0.2 \omega_{c i}$ for $v_{E} / v_{t i} \approx 1$. Whereas the Doppler shift is typically greater than $1.5 \omega_{c i}$ in previous reports of upshifting IEDD waves (Amatucci et al., 1996; 1998), the maximum Doppler shift here is $0.2 \omega_{c i}$. Measurements indicate that $\omega_{1}$ is less than $\omega_{c i}$ and in the neighborhood of $0.9 \omega_{c i}$, as estimated from $1.14 \omega_{c i}-0.2 \omega_{c i}$. We know that $\omega_{r} \omega_{1}$ is a positive quantity in the limit of zero $v_{E}$. We know that $\omega_{r} \omega_{1}$ does not change sign during the transition by virtue of $\left(\partial \omega_{1} / \partial v_{E}\right) v_{E},\left(\partial \omega_{r} / \partial v_{E}\right) v_{E}$, and $k_{\theta} v_{E}$ all being much less than $\omega_{1}$. Thus, $\omega_{r} \omega_{1}>0$ for both eigenmodes throughout the transition, even when the eigenmodes are predominantly reactive.

These results can be explained using aspects of inhomogeneous energy-density driven waves. Specifically, two reactive roots of the dispersion condition were found that had $\partial \omega_{r} / \partial v_{E}>0$, consistent with the observed upshift. However, the predicted growth rate is near zero (marginally stable) for a wide range of $v_{E}$ values. For these eigenmodes, $\omega_{1}<\omega_{c i}$ and $\partial \omega_{1} / \partial v_{E}<0$ so that as $k_{\theta}$ increases, the cyclotron damping decreases, consistent with the appearance of these modes. A thorough analysis of the dispersion condition confirms that predominantly reactive eigenmodes with $\omega_{r} \omega_{1}>0$ are possible, as the experiment demonstrates. An important point that can be made regarding the experimental observation of these eigenmodes is that the previous criteria identifying a predominantly reactive eigenmode should be modified so that cases in which $\omega_{r} \omega_{1}>0$, usually identified as predominantly dissipative, are not excluded. Since the value of $\left(k_{\theta} \rho_{i}\right)^{2}$ is so small for these observed predominantly reactive eigenmodes, the usual expansions in the dispersion function are not valid. Recall that the comparison of phase velocity with electron drift velocity remains the pri- 
mary distinction between predominantly dissipative and predominantly reactive eigenmodes.

\section{Conclusion}

A resonant to non-resonant transition in phase velocity is observed, corresponding to a transition from waves predominantly driven by the dissipative response of the IEDD mechanism to waves predominantly driven by the reactive response of the IEDD mechanism. Because $\omega_{r} \omega_{1}>0$ throughout the transition for one of the eigenmodes, this transition in phase velocity reflects unique aspects of IEDD waves compared to previous observations of IEDD waves. The fact that IEDD waves were observed counter-propagating along the magnetic field in the presence of a significant electron drift, means not only that the electron drift is not the primary source of free energy for these eigenmodes, but that these eigenmodes are robust to significant electron Landau damping. These results demonstrate that sheared plasma flow can play a dominant role in the generation of ion-cyclotron waves for conditions previously viewed as being incompatible with ion-cyclotron waves. The ramifications of these results extend to the interpretation of broadband and narrowband electrostatic ion-cyclotron waves in the ionosphere and magnetosphere where inhomogeneities in the perpendicular plasma flow is known to exist.

Acknowledgements. Useful discussions on the IEDD waveexcitation mechanism with G. Ganguli are gratefully acknowledged. This work is supported by the Office of Naval Research, the National Science Foundation, and NASA.

\section{References}

Amatucci, W. E., Walker, D. N., Antoniades, J. A., Ganguli, G., Duncan, D., Bowles, J. H., Gavrishchaka, V., and Koepke, M. E.: Plasma response to strongly sheared flow, Phys. Rev. Lett. 77, 1978-1981, 1996.

Amatucci, W. E., Walker, D. N., Ganguli, G., Duncan, D., Antoniades, J. A., Bowles, J. H., Gavrishchaka, V., and Koepke, M. E.: Velocity-shear-driven ion-cyclotron waves and associated transverse ion heating, J. Geophys. Res., 103, 11 711-11 724, 1998.

André, M., Behlke, R., Wahlund, J.-E., et al.: Multi-spacecraft observations of broadband waves near the lower hybrid frequency at the Earthward edge of the magnetosphere, Ann. Geophysicae, 19, 1471-1481, 2002.

Basu, S., Basu, S., MacKenzie, E., Fougere, P. F., Coley, W. R., Maynard, N. C., Winningham, J. D., Sugiura, M., Hanson, W. B., and Hoegy, W. R.: Simultaneous density and electric field fluctuation spectra associated with velocity shears in the auroral oval, J. Geophys. Res., 93, 115-136, 1984.

Bering, E. A.: The plasma environment of an auroral arc: Electrostatic ion cyclotron waves in the diffuse aurora, J. Geophys. Res., 89, 1635-1649, 1984.

Bonnell, J.: Identification of broadband ELF waves observed during transverse ion acceleration in the auroral ionsophere, $\mathrm{PhD}$ dissertation, Dep. of Electr. Eng., Cornell Univ., Ithaca, NY. 1997.
Bonnell, J., Kintner, P., Wahlund, J.-E., Lynch, K., and Arnoldy, R.: Interferometric determination of broadband ELF wave phase velocity within a region of transverse auroral ion acceleration, Geophys. Res. Lett., 23, 3297-3300, 1996.

Carroll, J. J., Koepke, M. E., Amatucci, W. E., Sheridan, T. E., and Alport, M. J.: A segmented disk electrode to produce and control parallel and transverse particle drifts in a cylindrical plasma, Rev. Sci. Instrum., 65, 2991-2995, 1994.

Carroll, J. J., Koepke, M. E., Zintl, M. W., Gavrishchaka, V., and Ganguli, G.: Velocity-shear origin of low-frequency electrostatic waves, Geophys. Res. Lett., 25, 3099-3102, 1998.

Ganguli, G., Lee, Y. C., and Palmadesso, P.: Electrostatic ioncyclotron instability due to a nonuniform electric field perpendicular to the external magnetic field, Phys. Fluids, 28, 761-763, 1985.

Ganguli, G., Lee, Y. C., and Palmadesso, P. J.: Kinetic theory for electrostatic waves due to transverse velocity shears, Phys. Fluids, 31, 823-838, 1988.

Ganguli, G., Lee, Y. C., Palmadesso, P. J., and Ossakow, S. L.: Oscillations in a plasma with parallel currents and transverse velocity shears, in: Physics of Space Plasmas (1988), (Eds) Chang, T., Crew, G. B., and Jasperse, J. R., Scientific Publishers, Cambridge, MA, 231-242, 1989.

Ganguli, G., Keskinen, M. J., Romero, H., Heelis, R., Moore, T., and Pollock, C.: Coupling of microprocesses and macroprocesses due to velocity shear: An application to the low-altitude ionosphere, J. Geophys. Res., 99, 8873-8889, 1994.

Gavrishchaka, V., Koepke, M. E., and Ganguli, G.: Dissipative properties of a magnetized plasma with a field-aligned drift and inhomogeneous transverse flow, Phys. Plasmas, 3, 3091-3106, 1996.

Hamrin, M., André, M., Ganguli, G., et al.: Inhomogeneous transverse electric fields and wave generation in the auroral region: A statistical study, J. Geophys. Res., 106, 10 803-10 816, 2001.

Hauck, J. P., Böhmer, H., Rynn, N., and Benford, G.: Ion beam excitation of ion-cyclotron wave and ion heating in plasmas with drifting electrons, J. Plasma Phys., 19, 253-265, 1978.

Kelley, M. C. and Carlson, C. W.: Observations of intense velocity shear and associated electrostatic waves near an auroral arc, J. Geophys. Res., 82, 2343-2348, 1977.

Kintner, P. M., Kelley, M. C., and Mozer, F. S.: Electrostatic hydrogen cyclotron waves near one earth radius altitude in the polar magnetosphere, Geophys. Res. Lett., 5, 139-142, 1978.

Kintner, P. M., Kelley, M. C., Sharp, R. D., Ghielmetti, A. G., Temerin, M., Cattell, C., Mizera, P. F., and Fennell, J. F.: Simultaneous observations of energetic (keV) upstreaming ions and electrostatic hydrogen cyclotron waves, J. Geophys. Res., 84, 7201-7212, 1979.

Kintner, P. M., Bonnell, J., Arnoldy, R., Lynch, K., Pollock, C., and Moore, T.: SCIFER: Transverse ion acceleration and plasma waves, Geophys. Res. Lett., 23, 1873-1876, 1996.

Kintner, P. M., Schuck, P. W., and Franz, J. R.: Spaced measurements and progress in understanding space plasmas waves, Phys. Plasmas, 7, 2135-2141, 2000a.

Kintner, P. M., Franz, J., Schuck, P., and Klatt, E.: Interferometric coherency determination of wavelength or what are broadband waves?, J. Geophys. Res., 105, 21 237-21 250, 2000 b.

Koepke, M. E., Amatucci, W. E., Carroll III, J. J., and Sheridan, T. E.: Experimental verification of the inhomogeneous energydensity driven instability, Phys. Rev. Lett., 72, 3355-3358, 1994.

Koepke, M. E., Amatucci, W. E., Carroll III, J. J., Gavrishchaka, V., and Ganguli, G.: Velocity-shear-induced ion-cyclotron tur- 
bulence: Laboratory identification and space applications, Phys. Plasmas, 2, 2523-2531, 1995.

Koepke, M. E., Carroll III, J. J., Zintl, M. W., Selcher, C. A., and Gavrishchaka, V.: Simultaneous observation of multiple nonlocal eigenmodes of an inhomogeneity-driven plasma instability, Phys. Rev. Lett., 78, 1441-1444, 1998a.

Koepke, M. E., Carroll III, J. J., and Zintl, M. W.: Excitation and propagation of electrostatic ion-cyclotron waves in plasma with structured transverse flow, Phys. Plasmas, 5, 1671-1680, 1998 b.

Koepke, M. E., Carroll III, J. J., and Zintl, M. W.: Laboratory simulation of broadband ELF waves in the auroral ionosphere, J. Geophys. Res., 104, 14397-14 415, 1999.

Lu, G., Reiff, P. H., Moore, T. E., and Heelis, R. A.: Upflowing ionospheric ions in the auroral region, J. Geophys. Res., 97, 16 855-16863, 1992.

Lund, E. J., Möbius, E., Klumpar, D. M., et al.: Direct comparison of transverse ion acceleration mechanisms in the auroral region at solar minimum, J. Geophys. Res., 104, 22 801-22 805, 1999.

Lynch, K. R., Arnoldy, R. L., Kintner, P. M., and Bonnell, J.: The AMICIST sounding rocket: A comparison of transverse ion acceleration mechanisms, Geophys. Res. Lett., 23, 3293-3296, 1996.

Marklund, G., Blomberg, L., Falthhammer, C.-G., and Lundqvist, P. A.: On intense diverging electric fields associated with black aurora, Geophys. Res. Lett., 21, 1859-1862, 1994.

Moore, T. E., Chandler, M. O., Pollock, C. J., et al.: Plasma heating and flow in an auroral arc, J. Geophys. Res., 101, 5279-5297,
1996.

Mozer, F. S., Cattell, C. A., Hudson, M. K., Lysak, R. L., Temerin, M., and Torbert, R. B.: Space Sci. Rev., 27, 155, 1980.

Peñano, J. R., Ganguli, G., Amatucci, W. E., Walker, D. N., and Gavrishchaka, V.: Velocity shear driven instabilities in a rotating plasma layer, Phys. Plasmas, 5, 4377-4383, 1998.

Pollock, C. J., Chandler, M. O., Moore, T. E., Waite, Jr., J. H., Chappell, C. R., and Gurnett, D. A.: A survey of upwelling ion event characteristics, J. Geophys. Res., 95, 18 969-18 980, 1990.

Robinson, R. M., Bering, E. A., Vondrak, R. R., Anderson, H. R., and Clothier, P. A.: Simultaneous rocket and radar measurements of currents in an auroral arc, J. Geophys. Res., 86, 7703-7717, 1981.

Rynn, N. and D'Angelo, N.: Device for generating a low temperature, highly ionized cesium plasma, Rev. Sci. Instrum., 31, 13261333, 1960.

Teodorescu, C., Reynolds, E. W., and Koepke, M. E.: Observation of inverse ion-cyclotron damping induced by parallel-velocity shear, Phys. Rev. Lett., submitted, 2002.

Torbert, R. B. and Mozer, F. S.: Electrostatic shocks as the source of discrete auroral arcs, Geophys. Res. Lett., 5, 135-138, 1978.

Tsunoda, R. T., Livingson, R. C., Vickrey, J. F., Heelis, R. A., Hanson, W. B., Rich, F. J., and Bythrow, P. F.: Dayside observations of thermal-ion upwelling at $800 \mathrm{~km}$ altitude: An ionospheric signature of the cleft ion fountain, J. Geophys. Res., 94, $15277-$ $15290,1989$. 